\title{
3C-SiC Films on Si for MEMS Applications: Mechanical Properties
}

\author{
C. Locke ${ }^{1}$, G. Kravchenko², P. Waters², J. D. Reddy², K. Du, A. A. Volinsky², \\ C. L. Frewin ${ }^{1}$ and S. E. Saddow ${ }^{1}$ \\ ${ }^{1}$ Dept. of Electrical Engineering, USF, 4202 E. Fowler Ave. ENB118, Tampa, FL, 33620 (USA) \\ ${ }^{2}$ Dept. of Mechanical Engineering, USF, 4202 E. Fowler Ave. ENB118, Tampa, FL, 33620 (USA) \\ aclocke@mail.usf.edu
}

Keywords: MEMS, 3C-SiC, Young's modulus, hardness, heteroepitaxy, CVD

\begin{abstract}
Single crystal 3C-SiC films were grown on (100) and (111) Si substrate orientations in order to study the resulting mechanical properties of this material. In addition, poly-crystalline 3C$\mathrm{SiC}$ was also grown on (100)Si so that a comparison with monocrystaline 3C-SiC, also grown on (100)Si, could be made. The mechanical properties of single crystal and polycrystalline 3C-SiC films grown on $\mathrm{Si}$ substrates were measured by means of nanoindentation using a Berkovich diamond tip. These results indicate that polycrystalline $\mathrm{SiC}$ thin films are attractive for MEMS applications when compared with the single crystal $3 \mathrm{C}-\mathrm{SiC}$, which is promising since growing single crystal 3C-SiC films is more challenging. MEMS cantilevers and membranes fabricated from a $2 \mu \mathrm{m}$ thick single crystal 3C-SiC grown on (100)Si under similar conditions resulted in a small degree of bow with only $9 \mu \mathrm{m}$ of deflection for a cantilever of $700 \mu \mathrm{m}$ length with an estimated tensile film stress of $300 \mathrm{MPa}$. Single crystal 3C-SiC films on (111)Si substrates have the highest elastic and plastic properties, although due to high residual stress they tend to crack and delaminate.
\end{abstract}

\section{Introduction}

There is intense interest in developing robust micro-electrical-mechanical systems (MEMS) for harsh applications such as chemical and biological sensors, advanced space actuators, etc. While silicon has been the primary material for MEMS applications, limitations in the temperature of operation of this material and the lack of a chemically inert surface has resulted in the development of alternate materials for these challenging applications [1]. The high hardness and chemical inertness of $\mathrm{SiC}$, coupled with its very high operating temperatures, has resulted in a high level of interest in developing SiC MEMS devices, with numerous groups around the world reporting progress with both the hexagonal and cubic polytypes of $\mathrm{SiC}[2,3]$. However, the ability to grow the cubic form of $\mathrm{SiC}$, namely $3 \mathrm{C}-\mathrm{SiC}$, on $\mathrm{Si}$ offers both a low-cost alternative for $\mathrm{SiC}$ MEMS coupled with the prospect to use well developed Si micromachining technology and processing to realize advanced, highly robust SiC MEMS devices [4].

While $\mathrm{SiC}$ is potentially an ideal material for power MEMS applications, deposition and processing techniques must be mastered before a functioning device can actually be built. It is advantageous to deposit $\mathrm{SiC}$ on $\mathrm{Si}$ wafers due to their high quality and low cost, in comparison to $\mathrm{SiC}$ substrates [5]. However, one has to overcome the $\sim 22 \%$ lattice mismatch between the 3C-SiC film and the (100) Si substrate, and after growth the $8 \%$ mismatch in thermal expansion [6]. Any temperature variation will cause additional stress. Also, 3C-SiC films grown on (111) Si wafers have a larger residual stress than films grown on (100). 3C-SiC can be hetero-epitaxially grown on a highly defective $\mathrm{SiC}$ buffer layers formed during the carbonization step.

\section{Film growth}

Single crystal (3C-SiC) and polycrystalline (poly-3C-SiC) SiC samples were grown on $\mathrm{Si}$ in order to study and compare the mechanical properties of the grown films. The samples were grown heteroepitaxially via chemical vapor deposition (CVD) in a hot-wall reactor [7]. Two sets of experiments were performed. The first was to compare single-crystal with polycrystalline 3C-SiC 
and these experiments. The film thickness of these samples was around 1-2 $\mu \mathrm{m}$. The orientation of the single crystal SiC film was both (100) and (111), corresponding to the Si substrate orientation. The second set of experiments was conducted to assess the uniformity of the grown films over a 50 $\mathrm{mm}$ wafer diameter. For these second experiments additional analysis, via evaluation of the wafer bow induced by the grown film, was conducted in order to estimate the residual stress in the films. In addition a $2 \mu \mathrm{m}$ 3C-SiC film grown on a $50 \mathrm{~mm}$ diameter (100)Si substrate was micromachined in order to assess the film stress, however this research is the subject of another paper in this conference [8]; therefore a comparison of this research with the present mechanical properties is made to provide a more comprehensive view of the perspective for using the films analyzed here for advanced MEMS applications.

Table 1 Single and poly crystalline 3C-SiC sample parameters

\begin{tabular}{|c|c|c|c|c|c|c|c|}
\hline \multirow[t]{2}{*}{ Film Type } & \multirow[t]{2}{*}{ Substrate } & \multicolumn{2}{|c|}{ Carbonization } & \multicolumn{2}{|l|}{ Growth } & \multirow{2}{*}{$\begin{array}{l}\text { Thickness }{ }^{1)} \\
{[\mu \mathrm{m}]}\end{array}$} & \multirow{2}{*}{$\begin{array}{l}\mathrm{RC} \mathrm{FWHM}^{2)} \\
\text { [arcsec] }\end{array}$} \\
\hline & & $\mathrm{P}$ [Torr] & $\mathrm{T}\left[{ }^{\circ} \mathrm{C}\right]$ & $\mathrm{P}$ [Torr] & $\mathrm{Si} / \mathrm{C}$ & & \\
\hline $3 \mathrm{C}-\mathrm{SiC}^{3)}$ & $\overline{(100)}$ & 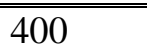 & 1135 & 100 & 1.13 & 2.3 & N/A \\
\hline $3 \mathrm{C}-\mathrm{SiC}^{3)}$ & (111) & 400 & 1135 & 100 & 1.3 & 2.5 & N/A \\
\hline Poly $^{3)}$ & (100) & 400 & 1135 & 100 & 1.2 & 2.4 & N/A \\
\hline $3 \mathrm{C}-\mathrm{SiC}^{4)}$ & (100) & 400 & 1135 & 100 & 1.13 & 1.9 & $2 \sim 300$ \\
\hline $3 \mathrm{C}-\mathrm{SiC}^{4)}$ & (111) & 400 & 1135 & 100 & 1.15 & 2.5 & $\sim 300$ \\
\hline
\end{tabular}

Notes: 1) Measured in wafer center via FTIR, 2) Measured with Philips X'pert diffractometer

3) Film grown on a $8 \mathrm{~mm}$ x $10 \mathrm{~mm}$ die, 4) Film grown on $50 \mathrm{~mm}$ wafer and measured as per [10].

3C-SiC single crystal films were grown on a $50 \mathrm{~mm}$ diameter Si wafers using a hot-wall CVD process without wafer rotation. Details of both the reactor and the growth process can be found elsewhere [9]. The 3C-SiC on $\mathrm{Si}$ deposition process was developed using the two step carbonization and growth method. $\mathrm{C}_{3} \mathrm{H}_{8}$ and $\mathrm{SiH}_{4}$ were used as the precursor gases to provide carbon and silicon, respectively. Ultra-high purity hydrogen, purified in a palladium diffusion cell, was employed as the carrier gas. Prior to growth, the Si substrates were prepared using the standard RCA cleaning method, followed by a 30 second immersion in diluted hydrofluoric acid to remove surface contaminants and the surface oxide. The first stage of the process, known as the carbonization step, involved heating the Si substrate from room temperature to the $1135^{\circ} \mathrm{C}$ carbonization temperature. The temperature was then maintained for two minutes to carbonize the substrate surface. After carbonization, $\mathrm{SiH}_{4}$ was introduced into the gas stream and the temperature was increased to the growth temperature. Using this process, a $2 \mu \mathrm{m}$ thick $3 \mathrm{C}-\mathrm{SiC}$ film was grown. For the $3 \mathrm{C}-\mathrm{SiC}$ films grown on (100)Si, the growth step was conducted at a process pressure of 100 Torr while the films grown on (111)Si was conducted at 400 Torr. After the growth process was completed, the wafer was cooled to room temperature in an Ar atmosphere. After deposition the film thickness was measured by Fourier transform infrared reflectance spectroscopy (FTIR) and confirmed by crosssection scanning electron microscopy analysis. The crystal orientation of the grown film was determined by X-ray diffraction (XRD) using a Philips X-Pert X-ray diffractometer. XRD data confirmed that the films were single crystal with an X-ray rocking curve FWHM of approximately 300 arcsec for the (100) and (111) orientations. Details of these films are presented in Table 1.

Polycrystalline growth follows the same procedure as single crystal growth on (100) Si with the exception of a higher flux of the growth species. The process conditions for the samples studied here were therefore identical to those listed above except that the $\mathrm{SiH}_{4}$ and $\mathrm{C}_{3} \mathrm{H}_{8}$ mass flow rates were 300 and $8.5 \mathrm{sccm}$, respectively. This process resulted in a poly-3C-SiC film with a thickness of approximately $4 \mu \mathrm{m}$. This film was then thinned so that the thickness was comparable to the single crystal films. The sample was polished on a polishing pad using $1 \mu \mathrm{m}$ diamond paste to both thin down the film thickness to match the single crystal film thickness and to smooth out the film surface roughness; the resulting film thickness was equivalent to the single crystal SiC film (1-2 $\mu \mathrm{m})$. 


\section{Mechanical Properties}

In the case of 3C-SiC films grown on (111)Si, a significant degree of wafer bow was present and visible to the naked eye. A comparison of two films grown with comparable processes as described above, also on $50 \mathrm{~mm}$ wafers, where the wafer bow was measured with an optical microscope and the stress calculated using the modified Stoney equation as performed previously on our films by Volinsky et. al. [10].This allowed a direct comparison of (100) and (111) oriented films grown on 2 inch wafer. For a film thickness of $\sim 2 \mu \mathrm{m}$ the radius of curvature was 15625 and $2084 \mathrm{~mm}$ resulting in a calculate tensile stress of 0.27 and $2.05 \mathrm{GPa}$ for these orientations, respectively. For the nonuniform (approximately 1-4 $\mu \mathrm{m}$ thick) 3C-SiC film on a $50 \mathrm{~mm} \mathrm{Si}$ wafer the film stress varies from 0.2 to $0.4 \mathrm{GPa}$ (higher on the thinner side).

Nanoindentation experiments were performed using a Hysitron Triboindenter. A Berkovich indenter was used to perform all indentation tests. Load controlled indentations were done to determine the elastic modulus and hardness of the films. Details of the technique and experimental procedure as applied to $\mathrm{SiC}$ films are in the literature [11]. Prior to measurement the samples were cleaved and glued to the sample holders using super glue and placed on the indenter stage for measurements. Each indent was performed individually after scanning the sample surface to ensure its smoothness. The low load transducer (maximum load of $10 \mathrm{mN}$ ) was used to determine the elastic modulus and hardness of the $3 \mathrm{C}$-SiC films. The experiment was carried out at loads varying from 0.5 to $10 \mathrm{mN}$. To determine the fracture toughness $(\mathrm{K})$, the low load transducer was replaced with a high load transducer. The above mentioned indentation procedure was followed at higher loads ranging from 100 to $1500 \mathrm{mN}$. The resulting measured mechanical properties are listed in Table 2.

Table 2. Measured SiC mechanical properties. ${ }^{1)}$

\begin{tabular}{lcll}
\hline \hline Material Type & Hardness [GPa] & Elastic Modulus [GPa] & Fracture toughness $\left[\mathrm{MPa} \cdot \mathrm{m}^{1 / 2}\right]^{2)}$ \\
\hline (100)Si $\mathrm{Si}$ & $12.46 \pm 0.78$ & $172.13 \pm 7.76$ & $1.59 \pm 0.21$ \\
Lely platelet 15R-SiC & $42.76 \pm 1.19$ & $442 \pm 16.34$ & $1.54 \pm 0.28$ \\
3C-SiC on $(100) \mathrm{Si}$ & $31.198 \pm 3.7$ & $433 \pm 50$ & 4.6 \\
3C-SiC on $(111) \mathrm{Si}$ & $>50$ & $>500$ & $\mathrm{NA}$ \\
Poly-3C-SiC on $(100) \mathrm{Si}$ & $33.54 \pm 3.3$ & $457 \pm 50$ & 2.18 \\
\hline \hline
\end{tabular}

1) Measured via nanoindentation using a Hysitron Triboindenter with a Berkovich indenter tip.

2) Crack lengths used to calculate the film fracture toughness using equation (1) from Volinsky et. al. [10].

Reported are the average values of the measured hardness and modulus of elasticity where at least five indents were performed at each varying maximum load. 15R-SiC was used as a comparison since this material is known to have a minimum amount of dislocations. There is a relatively large absolute variation in measured elastic modulus $( \pm 50 \mathrm{GPa})$, not typically observed in indentation of softer materials. One has to consider the relative variation, which is typically on the order of $10 \%$. The reported mechanical properties are for a maximum indentation depth range between 60 and $250 \mathrm{~nm}$, where quartz calibration is reasonable. Wear tests on the single crystal 3C$\mathrm{SiC}$ film were also performed in a $3 \times 3 \mathrm{~m}^{2}$ area using the low load transducer at $2 \mu \mathrm{N}$ normal load and $1 \mathrm{~Hz}$ frequency. After a number of wear cycles the scan area was zoomed to $5 \mathrm{x} 5 \mu \mathrm{m}$ to determine the wear. Almost no material wear was observed after 1000 wear cycles.

Load-controlled indentations were performed by varying maximum load, ranging from 0.5 to 10 $\mathrm{mN}$. The load-displacement curves exhibited by each type of 3C-SiC material may be found in [11]. The hardest material of the two samples had less penetration depth of the tip into the sample surface and hence the graph showed less displacement for the same amount of load applied. From this analysis it can be inferred that at lower load both the single and polycrystalline samples made elastic contact with the diamond probe. At higher loads, varying from 5 to $10 \mathrm{mN}$, plastic deformation was observed in the film. For indentation performed at a load of $10 \mathrm{mN}$ it was observed that the indenter penetrated more into the single crystal 3C-SiC film surface compared to that of the polycrystalline 3C-SiC film on (100)Si substrate. 3C-SiC films grown on (111)Si have 
superior mechanical properties, although due to high residual stress they produce large wafer bow and tend to crack and delaminate..

At higher loads, cracks were observed along the sharp corners of the Berkovich tip and the crack length was used to calculate the film fracture toughness using equation (1) from reference [11]. Table 2 also gives the fracture toughness values. The cause for the low fracture toughness in this case compared to the bulk values is due to the tip penetrating into the Si substrate and substrate fracture skewing the results to a lower substrate fracture toughness value. Continuous wear tests were performed on the polycrystalline 3C-SiC surface showed very little or negligible wear, as only 1-2 $\mathrm{nm}$ of material depth was removed. This result confirms the high wear resistance of 3C-SiC films necessary for MEMS applications.

\section{Acknowledgement}

3C-SiC growth in S.E. Saddow's laboratory was supported by the Army Research Laboratory under Grant No. DAAD19-R-0017 (B. Geil). Alex Volinsky would like to acknowledge support from NSF under CMMI contracts 0631526, 0600266 and 0600231.

\section{References}

[1] M. Mehregany et al.: Thin Solid Films Vol. 355-356 (1999), p. 518

[2] M. Mehregany et al.: Proc. of the IEEE Vol. 86 (1998), p. 1594

[3] P. M. Sarro: Sensors and Actuators A: Physical Vol. 82 (2000), p. 210

[4] M. Reyes et al.: Mat Sci Forum Vol. 527-529 (2006), p. 191

[5] S. Nishino et al.: Appl. Phys. Lett. Vol. 42 (1983), p. 460

[6] A. Severino et al.: J. Appl. Phys. Vol. 102 (2007), p. 023518

[7] R. L. Myers et al.: J. Crystal Growth Vol. $284-285$ (2005), p. 483

[8] M. Mehregany et al.: IEEE Trans. Electron Dev. Vol. 44 (1997), p. 74

[9] R. Anzalone et al.: ibid

[10] M. Reyes et al.: Mat. Res. Soc. Symp. Proc. Vol. 911 (2006), p. 79

[11] A. A. Volinsky et al.: Mat. Res. Soc. Symp. Proc. Vol. 1069 (2008)

[12] J. D. Reddy et al.: Mat. Res. Soc. Symp. Proc. Vol. 1069 (2008) 
Silicon Carbide and Related Materials 2008

doi:10.4028/www.scientific.net/MSF.615-617

3C-SiC Films on Si for MEMS Applications: Mechanical Properties doi:10.4028/www.scientific.net/MSF.615-617.633

\section{References}

[1] M. Mehregany et al.: Thin Solid Films Vol. 355-356 (1999), p. 518 doi:10.1016/S0257-8972(99)00374-6

[2] M. Mehregany et al.: Proc. of the IEEE Vol. 86 (1998), p. 1594 doi:10.1109/5.704265

[3] P. M. Sarro: Sensors and Actuators A: Physical Vol. 82 (2000), p. 210 doi:10.1016/S0924-4247(99)00335-0

[4] M. Reyes et al.: Mat Sci Forum Vol. 527-529 (2006), p. 191 doi:10.4028/www.scientific.net/MSF.527-529.307

[5] S. Nishino et al.: Appl. Phys. Lett. Vol. 42 (1983), p. 460 doi:10.1063/1.93970

[6] A. Severino et al.: J. Appl. Phys. Vol. 102 (2007), p. 023518 doi:10.1063/1.2756620

[7] R. L. Myers et al.: J. Crystal Growth Vol. 284-285 (2005), p. 483

[8] M. Mehregany et al.: IEEE Trans. Electron Dev. Vol. 44 (1997), p. 74 doi:10.1109/16.554795

[9] R. Anzalone et al.: ibid

[10] M. Reyes et al.: Mat. Res. Soc. Symp. Proc. Vol. 911 (2006), p. 79

[11] A. A. Volinsky et al.: Mat. Res. Soc. Symp. Proc. Vol. 1069 (2008)

[12] J. D. Reddy et al.: Mat. Res. Soc. Symp. Proc. Vol. 1069 (2008) 